\title{
Reduction of Power Transformer Core Noise Generation due to Magnetostriction Induced Deformations using Fully Coupled Finite Element Modeling Optimization Procedures
}

\author{
Mingyong Liu ${ }^{1,2}$, Olivier Hubert ${ }^{2}$, Xavier Mininger ${ }^{1}$, Frédéric Bouillault ${ }^{1}$, Laurent Bernard ${ }^{3}$, and Thierry Waeckerlé ${ }^{4}$ \\ ${ }^{1}$ GeePs, CNRS UMR 8507, CentraleSupelec, Univ Paris Sud, UPMC, 91192 Gif-sur-Yvette, France \\ ${ }^{2}$ LMT-Cachan, CNRS UMR 8535, ENS-Cachan, Univ. Paris-Saclay, 94235 Cachan Cedex, France \\ ${ }^{3}$ GRUCAD/EEL/CTC, Universidade Federal de Santa Catarina, Florianpolis 88040-900, Brazil \\ ${ }^{4}$ Aperam alloys Imphy, 58160 Imphy, France
}

\begin{abstract}
This work focuses on the development of an algorithm for the prediction of transformer core deformation, using a fully coupled magneto-mechanical approach. An imposed magnetic flux method coupled with finite element modeling is employed for the magnetic resolution. The constitutive law of the material is considered with a simplified multi-scale model (SMSM) describing both magnetic and magnetostrictive anisotropies. Magnetostriction is introduced as an input free strain of a mechanical problem to get the deformation and displacement fields. A 3D estimation of acoustic power is carried out to evaluate the global core deformation and acoustic noise level. The numerical process is applied to a three-phase transformer made of Grain Oriented (GO) FeSi core, leading to a set of noise estimation for different flux excitations and core geometries.
\end{abstract}

Index Terms-Magnetostriction, power transformers, noise, iron-silicon alloys, finite element method.

\section{INTRODUCTION - OBJECTIVES}

$\mathbf{T}$ HE ELECTRIC power of aircraft onboard systems is increasing with the proliferation of comfort equipment and the gradual replacement of hydraulic actuators by electric actuators. As a consequence, higher power transformers are needed to feed the different electric devices. This evolution usually means bigger transformers and higher weight, leading to more noise emission which is related to their size and surface. There are two ways to make the transformer on aircraft more powerful but lightweight with relatively low noise emission. One way is to develop high-performance materials with high power density and low magnetostriction. Materials such as iron-cobalt alloys saturate at nearly $2.35 \mathrm{~T}$ [1]. They are ideal to increase the power density of the transformers. Unfortunately, iron-cobalt alloys generate high magnetostriction [2], [3]. Materials such as some iron-nickel alloys generate nearly no magnetostriction, but their highstress sensitivity [4] and low magnetic saturation strongly limit their use [5]. Another way proposed in this paper is to properly design the structure of transformer core, at a given material, offering the less global core deformation due to magnetostriction, and then noise emission.

The noise of transformer is mainly caused by the interactions between the transformers magnetic stray field and the current-carrying winding loops [6] and also by periodic deformations of sheets of the transformer core. This deformation has two origins: i) elastic strain associated with magnetic forces appearing on the free surface and volume; ii) spontaneous magnetostriction depending on the local magnetic state of the material [7]. Studies have shown, on the other hand, that the junction of the transformer core pieces strongly contributes to the noise generation [8]. For the application of small transformers in the aircraft considered in this paper, core sheets are preferred in the form of 'E' and 'I', or ideally noncut in the form of ' 8 '. Since there is only little air-gap between each electrical sheet of the transformer core, magnetic forces are negligible [9]. Core deformation due to magnetostriction is then considered as the main source of noise emission.

This work proposes a complete modeling chain of such a transformer, from the magnetic flux excitation to the noise estimation. Other mechanisms coming from assembly precision and air gaps may also have an influence on the sound emission but are not considered in this modeling chain. In section II a state of the art is presented dedicated to the recent works in this field. In section III] a fully coupled simplified multiscale model (SMSM) is introduced, describing both magnetic and magnetostrictive anisotropy. In section IV, the structure of studied stacked transformer core is described, followed by a brief recall of the homogenization strategy, leading to a 2D modeling of a multi-layer transformer. In section $\mathrm{V}$, the global modeling strategy is introduced, including the magnetostatic problem solving, the mechanical feedback loop, the equivalent force computation, the elastodynamic modeling and the acoustic power estimation. By imposing directly the magnetic flux in limbs, displacement fields and acoustic power estimation of the transformer core are finally obtained. In section VI. GO FeSi alloy with strong anisotropy is chosen to test this modeling chain because of its high permeability and low magnetostriction in the easy magnetization direction. Applications to an '8-shaped' stacked core made of GO FeSi sheet in the form of ' $E$ ' and 'I' are carried out as examples. In section VII a set of computations is carried out with different geometries keeping the same mass and winding sections. A map of acoustic power is finally obtained, leading to design guidelines that offer higher power density at a lower noise 
level.

\section{STATE OF THE ART}

Recently, magnetostriction induced vibration on electric devices such as electric machines [10], [11] and inductors [12], has received more and more attention. Studies of magnetostriction induced vibration and noise on power transformers are numerous: Mizokami et al. [13] demonstrate that the compression stress along rolling direction of the electrical steel increases magnetostriction and noise level. Zhu et al. [14] find that filling the multi-joint gaps with nanocrystalline soft magnetic composite material decreases magnetostriction and vibration of the core because of an improved distribution of magnetic flux. Hsu et al. [15] propose a new method to reduce the transformer core noise by re-arranging the step-lapped joint structure. Efforts are made to study the correlation of magnetostriction variation [16], DC bias [17], magnetostriction force spectrum [18], harmonic voltages [19], climbings [20] and magnetic hysteresis [21] on power transformer noise emission.

In terms of experimentation, vibration and deformation of a test transformer core are measured by means of accelerometers which give the acceleration of certain points [17], or by means of a laser scanning vibrometer, giving a 3D field of surface speed [22], [23]. Both need to consider the suspension of the core and post-processing of the measurement to correct the rigid body movement [9]. Direct measurement of noise is also carried out, allowing the comparison of different transformer cores [24] and the study of the physical parameters' influences, leading to an experience deduced noise computation equation [25].

In terms of modeling, efforts are made to estimate magnetostriction induced deformation, vibration and noise emission of the power transformer core. Hu et al. [26] give a complete finite element analysis modeling chain from current injection to the noise emission. Shuai et al. [23] push it even further to the optimization step, taking efficiency and vibration as criterions. But both of them use an isotropic and linear material model, which strongly limits the accuracy of their computation. Similar studies [27], [28], [29] are carried out by implementing an anisotropic, nonlinear and straindependant material model to simulate the vibration due to magnetostriction. However, the magnetic circuits they worked on seem to be far from a laminated transformer core.

Despite strong recent efforts in this field, no commercial software nor academic code is yet available to estimate and optimize the global deformation or noise level of a laminated transformer core, with the consideration of material anisotropy, nonlinearity of the magneto-mechanical behavior and stress dependence. They are the motivations of this work.

This article is based on our previous work [9], briefly recalled hereafter. It dealt with the prediction of the deformation of a multilayer transformer stacked 'E-I' core. The problem was solved by a stepping sequential approach: first magnetic resolution with current excitation, second mechanical resolution. A weakly coupled SMSM describing both magnetic and magnetostrictive anisotropies was used as the constitutive law of the material. The transformer core structure was modeled in 2-D, and a homogenization technique was implemented to take the anisotropic behavior of each layer into consideration and define an average behavior. A three-layer transformer prototype was fabricated with $\mathrm{GO}$ FeSi. Good agreements were obtained by comparing the experimental measurements and the modeling results.

However, it is well known that the power of transformer is related to the excitation tension, proportional to magnetic flux. In the previous modeling chain, the current was imposed, making difficult the comparison of different core structures at the same power level. It was consequently hard to tell which core structure generates less noise only by looking at the strain distribution. Moreover, the deformation induced stress has an impact on the material properties, which also needs proper studies. The stress dependence of magnetic material is non-negligible, especially for 'E-I' stacked core where layers tend to deform in different ways introducing self-stress. The improvements proposed in this paper are finally the followings:

1) a feedback loop of magnetostriction induced stress is implemented, leading to fully coupled modeling chain with a more accurate prediction of the magneto-mechanical fields.

2) the magnetic flux is directly imposed in the limbs, facilitating core analysis and comparison at the same power level.

3) a block of post-processing is added, allowing the computation of acoustic power. This gives a new criterion for optimization of the core structure.

4) an optimization process is carried out for core dimensions, in order to minimize acoustic vibrations of an E-I transformer core with interleaved RD and TD layers.

These improvements authorise the optimization process of the transformer cores.

\section{Constitutive Law}

The constitutive law used for the modeling is a simplified version of a full multi-scale magneto-mechanical model (MSM) [30], [31]. In the complete version, the considered scales are the magnetic domain, the single crystal and the polycrystalline (macroscopic) scales. This model allows an accurate modeling of the anhysteretic magnetic and magnetostrictive behavior of ferro/ferrimagnetic materials, and takes the effect of mechanical stress into account. The number of internal variables of such model is nevertheless too high to be implemented in a complex structure model with many degrees of freedom. The simplified version (SMSM) has been proposed in [32] and developed in [9].

This SMSM considers a magnetic material (usually polycrystalline) as an equivalent single-crystal. This equivalent single crystal consists of magnetic domains oriented in different directions. Local magnetization of domain family $\alpha(1)$ is a vector of norm the magnetization saturation $M_{s}$ oriented in the direction $\vec{\gamma}^{\alpha}$.

$$
\vec{M}^{\alpha}=M_{s} \vec{\gamma}^{\alpha}
$$


Local free energy of a magnetic domain family $(\alpha)$ oriented in direction $\vec{\gamma}^{\alpha}$ is expressed as the sum of four contributions.

$$
W_{t o t}^{\alpha}=W_{m a g}^{\alpha}+W_{a n}^{\alpha}+W_{m e}^{\alpha}+W_{c o n f}^{\alpha}
$$

$W_{m a g}^{\alpha}$ is the Zeeman energy, introducing the effect of the applied magnetic field on the equilibrium state. $W_{m a q}^{\alpha}$ (equation 3 - where $\mu_{0}$ is the vacuum permeability) tends to align the local magnetization $\vec{M}^{\alpha}$ along the magnetic field $\vec{H}$ considered as homogeneous over the crystal.

$$
W_{m a g}^{\alpha}=-\mu_{0} \vec{M}^{\alpha} \cdot \vec{H}
$$

$W_{a n}^{\alpha}$ is an anisotropy energy term related to the crystallographic texture of the polycrystal and the magneto crystalline anisotropy (4). $K_{1}$ is a physical anisotropy constant representing the anisotropy level. $\mathbb{P}$ is a $4^{\text {th }}$ order tensor that describes the symmetries (cubic symmetry for $\mathrm{FeSi}$ ). $\boldsymbol{Q}$ is a simple transformation matrix from crystal frame (CF) to the sample frame (SF).

$$
W_{a n}^{\alpha}=K_{1}\left(\vec{\gamma}^{\alpha} \otimes \vec{\gamma}^{\alpha}\right):\left(\boldsymbol{Q} \cdot \boldsymbol{Q} \cdot \mathbb{P} \cdot{ }^{t} \boldsymbol{Q} \cdot{ }^{t} \boldsymbol{Q}\right):\left(\vec{\gamma}^{\alpha} \otimes \vec{\gamma}^{\alpha}\right)
$$

$W_{m e}^{\alpha}$ is the magneto-elastic energy (5). It is written as a function of the free magnetostriction strain $\epsilon_{\mu}^{\alpha}$ of the domain family $\alpha$ (given by equation (6) in the $\mathrm{CF}$ and equation (7) in the SF) and of the stress tensor $\sigma$ over the crystal. $\lambda_{100}$ (resp. $\lambda_{111}$ ) is the saturation magnetostriction strain along the direction $<100>$ (resp. $<111>$ ) of the single crystal. $\gamma_{1}$, $\gamma_{2}$ and $\gamma_{3}$ are the direction cosines of the magnetization vector in the CF.

$$
\begin{gathered}
W_{m e}^{\alpha}=-\boldsymbol{\sigma}: \boldsymbol{\epsilon}_{\mu}^{\alpha} \\
\boldsymbol{\epsilon}_{\mu C F}^{\alpha}=\frac{3}{2}\left(\begin{array}{ccc}
\lambda_{100}\left(\gamma_{1}^{2}-\frac{1}{3}\right) & \lambda_{111} \gamma_{1} \gamma_{2} & \lambda_{111} \gamma_{1} \gamma_{3} \\
\lambda_{111} \gamma_{1} \gamma_{2} & \lambda_{100}\left(\gamma_{2}^{2}-\frac{1}{3}\right) & \lambda_{111} \gamma_{2} \gamma_{3} \\
\lambda_{111} \gamma_{1} \gamma_{3} & \lambda_{111} \gamma_{2} \gamma_{3} & \lambda_{100}\left(\gamma_{3}^{2}-\frac{1}{3}\right)
\end{array}\right)_{(6)}{ }^{C F} \\
\boldsymbol{\epsilon}_{\mu}^{\alpha}=\boldsymbol{Q} \cdot \boldsymbol{\epsilon}_{\mu C F}^{\alpha} \cdot{ }^{t} \boldsymbol{Q}
\end{gathered}
$$

$W_{\text {conf }}^{\alpha}$ is a configuration energy term, which allows some peculiar initial distribution of domains (residual stress effect, demagnetizing surface effect...). Its simplified expression for a thin electrical sheet is expressed as in (8). $C$ is an adjusting constant that defines the maximum level of the configuration energy. $\gamma_{z}$ is the out-of-plane component of magnetic domain direction $\vec{\gamma}^{\alpha}$ in SF.

$$
W_{\text {conf }}^{\alpha}=C\left(\gamma_{z}\right)^{2}
$$

A Boltzmann like function is used to calculate the volume fraction $f_{\alpha}$ of domain $\alpha$ :

$$
f_{\alpha}=\frac{\exp \left(-A_{s} W_{t o t}^{\alpha}\right)}{\sum_{\alpha} \exp \left(-A_{s} W_{t o t}^{\alpha}\right)}
$$

where $A_{s}$ is an adjusting parameter related to the initial susceptibility. Macroscopic quantities are obtained by averaging the microscopic quantities over the single crystal volume (10). $\epsilon_{\mu}$ is the free magnetostrictive strain tensor at the macroscopic scale.

$$
\vec{M}=\sum_{\alpha} f_{\alpha} \vec{M}^{\alpha} \quad \text { and } \quad \epsilon_{\mu}=\sum_{\alpha} f_{\alpha} \epsilon_{\mu}^{\alpha}
$$

SMSM allows finally the computation of the macroscopic anhysteretic magnetostriction and magnetization at a given magnetic field level $\vec{H}$. We define on the other hand the differential magnetic susceptibility tensor $\chi$. It is given by (11) and can be derived from the SMSM as proposed in [33]. This is added in the SMSM as an output of the model, essential for the non-linear magnetic resolution using the Newton-Raphson method.

$$
\chi=\frac{\partial \vec{M}}{\partial \vec{H}}=\mu_{0} A_{s}\left(M_{s}^{2} \sum_{\alpha} f_{\alpha} \vec{\gamma}^{\alpha} \otimes \vec{\gamma}^{\alpha}-\vec{M} \otimes \vec{M}\right)
$$

\section{GeOMETRY OF STACKED TRANSFORMER CORE AND HOMOGENIZATION STRATEGY FOR 2D REPRESENTATION}
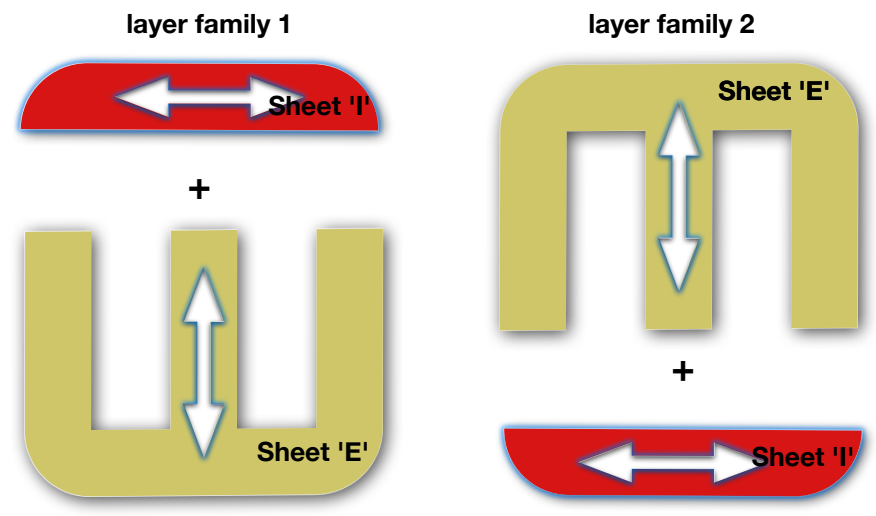

Fig. 1: Transformer core layers : layer family 1 (left) with 'Ishaped' sheet (red region) on top and 'E-shaped' sheet (yellow region) on bottom; layer family 2 (right) with 'E-shaped' sheet on top and 'I-shaped' sheet on bottom. White arrows indicate the easy magnetization direction.

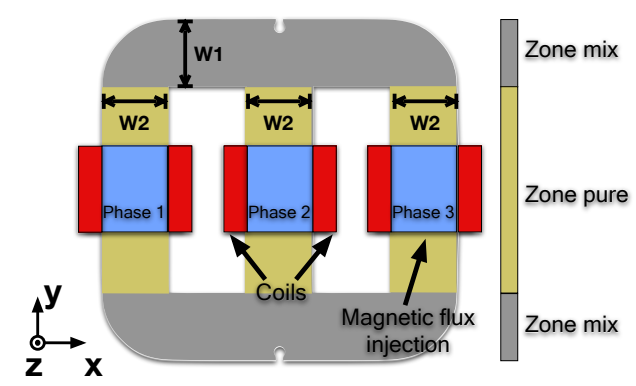

Fig. 2: Equivalent 2D model with coils and magnetic flux excitation.

A transformer stacked core is assembled with hundreds of layers. Typically, a layer is assembled anti-parallel to its neighbour layer, forming two layer families, each consisting of an 'E-shaped' sheet and an 'I-shaped' sheet, as illustrated in Fig $1 . f_{1}$ and $f_{2}$ define the section (or volume) fraction of layer family 1 ('E-shaped' sheet upside and 'I-shaped' sheet downside) and layer family 2 ('E-shaped' sheet downside and 'I-shaped' sheet upside) respectively. Since magnetic materials are often anisotropic, the easy magnetization directions of 'Eshaped' sheets and 'I-shaped' sheets are oriented differently, 
leading to inhomogeneous behavior from layer to layer (Fig 2). To take this z-axis inhomogeneity into account for 2D modeling, a homogenization strategy of a heterogeneous problem is required to extract the average behavior. The following assumptions are considered:

- Sheets are usually very thin, allowing to assume a 2D homogeneous magnetic field using the classical tangential magnetic field continuity condition.

- Sheets are considered perfectly stuck together, allowing to assume a homogeneous displacement field at the interface between two sheets. Homogeneous strain can consequently be considered in the sheet plane.

- The transformer is supposed mechanically unloaded and thin enough to consider out-of-plane stress free conditions on upper and lower surfaces.

- Elastic and magnetostrictive strains are considered sufficiently small to allow us an additive description (between elastic and magnetostrictive strains) of the total deformation.

With the mentioned magnetic and mechanical hypotheses, mixing rules $[12)-(16)$ are applied [9].

$$
\begin{gathered}
\vec{M}=f_{1} \vec{M}_{1}+f_{2} \vec{M}_{2} \\
\vec{B}=f_{1} \vec{B}_{1}+f_{2} \vec{B}_{2} \\
\chi=f_{1} \chi_{1}+f_{2} \chi_{2} \\
\boldsymbol{\epsilon}_{\mu}=f_{1} \boldsymbol{\epsilon}_{\mu 1}+f_{2} \boldsymbol{\epsilon}_{\mu 2} \\
\boldsymbol{\sigma}=f_{1} \boldsymbol{\sigma}_{1}+f_{2} \boldsymbol{\sigma}_{2}
\end{gathered}
$$

$f_{1}+f_{2}=1$, and in real transformer with hundreds of layers $f_{1} \approx f_{2} \approx 0.5$.

\section{Global modeling STRATEGY}

The global modeling strategy is summarized in Fig 3 This fully coupled magneto-mechanical problem is solved at two stages: magnetic resolution coupled with mechanical feedback loop using a quasi-static approach in the time domain in the first step, then harmonic mechanical resolution and acoustic power estimation in the frequency domain in a second step. Magnetic resolution is carried out with imposed magnetic flux. SMSM is then engaged to take into account anisotropy, nonlinearity and stress dependency of the material. An homogenization rule is then applied, leading to an average behaviour of the different family layers. At the end of each magnetic iteration, an equivalent force is computed from the free magnetostriction strain. The mechanical feedback loop is carried out in parallel with the magnetic resolution loop. Through the static mechanical resolution, the total deformation of the structure is computed, leading to induced strain in each family layer. This is injected into SMSM to adapt the material behaviour under stress. Harmonic mechanical resolution and acoustic power are carried out in the frequency domain to avoid the transient state and obtain better efficiency.

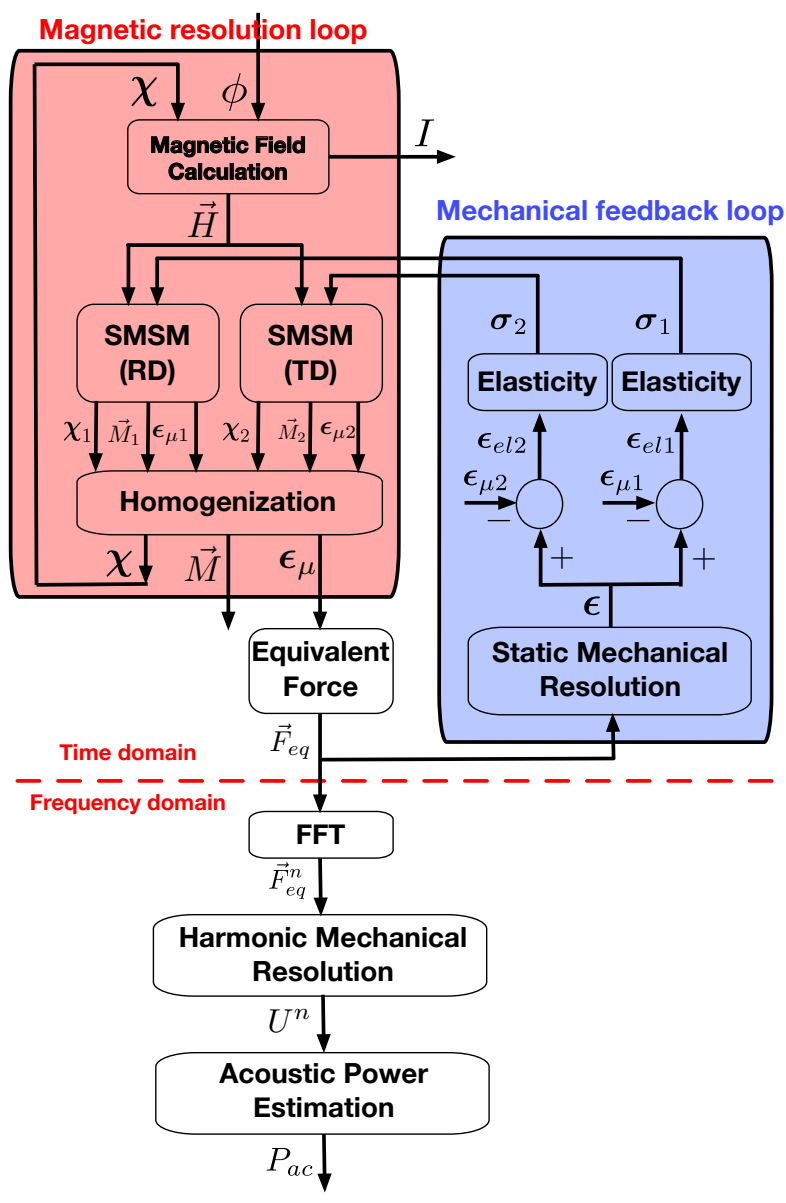

Fig. 3: Global modeling strategy.

\section{A. Magnetic resolution with imposed magnetic flux method}

One important criterion for power transformer design is the power-to-mass ratio (transmitted power per unit mass), which is proportional to the magnetic flux $\phi$ circulating in the transformer core. It is interesting to compare the core deformation and excitation current of different cores at the same imposed magnetic flux. Besides, transformers are normally driven with voltage, which is proportional to the magnetic flux in each phase. Imposing balanced magnetic flux often leads to unbalanced currents in the coils, because of the unbalanced reluctance in most of the transformer parts.

To simplify this problem, the modeling is carried out under magnetic quasi-static assumption (induced current not considered). Since the constitutive model is anhysteretic, magnetic hysteresis and dynamic behavior are not considered in this paper. The imposed magnetic flux is first discretized in the time domain. The magnetic resolution uses an iterative Newton-Raphson method: a magnetic flux $\phi$ is imposed; the magnetization $\vec{M}$ is arbitrarily defined at the first loop allowing a first estimation of the magnetic field $\vec{H}$. The differential magnetic susceptibility $\chi$ is then updated, using the SMSM and the homogenization rules. The procedure is iterated until convergence.

The following part gives some details on the magnetic field computation. A unit current potential vector $\vec{T}_{0}$ is defined to 
get $I \vec{\nabla} \times \vec{T}_{0}=\vec{j}$, with $\vec{j}$ and $I$ respectively the unknown current density and current amplitude in the coils. Combined with induction flux conservation equation $\operatorname{div}(\vec{B})=0$, the weak formulation is shown in (17). $\Omega$ is the integration domain, $v$ is a test function. $\mu_{0}$ and $\nu_{0}$ are respectively the vacuum permeability and reluctivity. $\varphi$ is the magnetic scalar potential, leading to the magnetic field computation $\vec{H}=-\vec{\nabla} \varphi+I \vec{T}_{0}$.

A second equation $(18)$ is obtained from the total magnetic energy formulation $\phi I=\int_{\Omega} I \overrightarrow{T_{0}} \cdot \vec{B} \mathrm{~d} \Omega$ [34]. The non-linear problem is solved at a given applied flux leading to current value $I$ in the coils and magnetic scalar potential $\varphi$.

$$
\left\{\begin{aligned}
\mathcal{F}_{1}(\varphi, I) & =\int_{\Omega} \vec{B} \cdot \vec{\nabla} v \mathrm{~d} \Omega=0 \\
& =\int_{\Omega} \mu_{0}\left(-\vec{\nabla} \varphi+I \vec{T}_{0}+\vec{M}\right) \cdot \vec{\nabla} v \mathrm{~d} \Omega \\
\mathcal{F}_{2}(\varphi, I) & =\int_{\Omega} \vec{T}_{0} \cdot \vec{B} \mathrm{~d} \Omega-\phi=0 \\
& =\int_{\Omega} \mu_{0}\left(-\vec{\nabla} \varphi+I \vec{T}_{0}+\vec{M}\right) \cdot \vec{T}_{0} \mathrm{~d} \Omega-\phi
\end{aligned}\right.
$$

At a given moment, this quasi-static non-linear magnetic problem is solved using the Newton-Raphson method. As a consequence, the problem defined by equation (17) and (18) is developed in first order Taylor series, where $i=1,2$ :

$\mathcal{F}_{i}(\varphi+\Delta \varphi, I+\Delta I)=\mathcal{F}_{i}(\varphi, I)+\frac{\partial \mathcal{F}_{i}}{\partial \varphi} \Delta \varphi+\frac{\partial \mathcal{F}_{i}}{\partial I} \Delta I=0$

this problem is then discretized, leading to the iterative relation 20p and 21). $\boldsymbol{\mu}_{r d}^{p}$ is the differential relative magnetic permeability tensor for the $p^{\text {th }}$ iteration, obtained from $\chi^{p}$ $\left(\boldsymbol{\mu}_{r d}^{p}=\chi^{p}+1\right) \cdot \Delta \varphi$ and $\Delta I$ give the difference between iterations $\left(\Delta \varphi=\varphi^{p+1}-\varphi^{p}, \Delta I=I^{p+1}-I^{p}\right)$.

$$
\left\{\begin{array}{l}
\quad \int_{\Omega} \mu_{0} \boldsymbol{\mu}_{r d}^{p} \cdot \vec{\nabla} v \cdot \vec{\nabla}(\Delta \varphi) \mathrm{d} \Omega \\
-\int_{\Omega} \mu_{0} \boldsymbol{\mu}_{r d}^{p} \cdot \vec{T}_{0} \cdot \vec{\nabla} v(\Delta I) \mathrm{d} \Omega \\
=\int_{\Omega} \vec{B}^{p} \cdot \vec{\nabla} v \mathrm{~d} \Omega \\
-\int_{\Omega} \mu_{0} \boldsymbol{\mu}_{r d}^{p} \cdot \vec{T}_{0} \cdot \vec{\nabla}(\Delta \varphi) \mathrm{d} \Omega \\
+\int_{\Omega} \mu_{0} \boldsymbol{\mu}_{r d}^{p} \cdot \vec{T}_{0} \cdot \vec{T}_{0}(\Delta I) \mathrm{d} \Omega \\
= \\
\phi-\int_{\Omega} \vec{B}^{p} \cdot \vec{T}_{0} \mathrm{~d} \Omega
\end{array}\right.
$$

The electromagnetic system constituted by 20) and 21, is extended in this paper to a three phase problem. Considering a three phase transformer in star configuration without neutral, it is possible to add a closure equation for current: $I_{1}+I_{2}+I_{3}=0$. (22) gives the new global weak formulation system of equations, with $m$ indicating the number of degrees of freedom. Components in (22) are defined in (23)-(27). $i$ and $j$ denote respectively the index of row and column of each component, and $k$ is the number of phases.

$$
\left(\begin{array}{c:cc}
\boldsymbol{a}_{m * m} & \boldsymbol{b}_{1}-\boldsymbol{b}_{3} \boldsymbol{b}_{2}-\boldsymbol{b}_{3} \\
\hdashline \boldsymbol{b}_{1}^{t}-\boldsymbol{b}_{3}^{t} & c_{1}-c_{3} & 0 \\
\boldsymbol{b}_{2}^{t}-\boldsymbol{b}_{3}^{t} & 0 & c_{2}-c_{3}
\end{array}\right) \cdot\left(\begin{array}{c}
\Delta \boldsymbol{\varphi}_{m * 1} \\
\hdashline I_{1}-\Delta I_{3} \\
\Delta I_{2}-\Delta I_{3}
\end{array}\right)=\left(\begin{array}{c}
\boldsymbol{d}_{m * 1} \\
e_{1}-e_{3} \\
e_{2}-e_{3}
\end{array}\right)
$$

Using an imposed magnetic flux computation at each phase $\phi_{k}$, two kinds of unknowns are solved at once: $\varphi$ is vector gathering the magnetic scalar potential values at the nodes; $I_{k}$ is the $k^{\text {th }}$ phase current.

$$
\begin{aligned}
a^{i j} & =\iint_{\Omega} \mu_{0} \boldsymbol{\mu}_{r d} \cdot \vec{\nabla} \varphi^{i} \cdot \vec{\nabla} v^{j} \mathrm{~d} \Omega \\
b_{k}^{i} & =-\iint_{\Omega} \mu_{0} \boldsymbol{\mu}_{r d} \cdot \vec{\nabla} v^{i} \cdot \vec{T}_{0(k)} \mathrm{d} \Omega \\
c_{k} & =\iint_{\Omega} \mu_{0} \vec{T}_{0(k)} \cdot \vec{T}_{0(k)} \mathrm{d} \Omega \\
d^{i} & =\iint_{\Omega} \vec{\nabla} v^{i} \cdot \vec{B}^{i} \mathrm{~d} \Omega \\
e_{k} & =\phi_{k}-\iint_{\Omega} \vec{B} \cdot \vec{T}_{0(k)} \mathrm{d} \Omega
\end{aligned}
$$

After resolution of this non-linear electro-magnetic system, $I_{k}$ and $\varphi$ are obtained by accumulating $\Delta I_{k}$ and $\Delta \varphi \cdot \vec{M}$ and $\epsilon_{\mu}$ field are the output of the SMSM at each time step.

\section{B. Equivalent force}

The free magnetostrictive strain $\epsilon_{\mu}$ computed from the SMSM is then transformed into a nodal equivalent force $\vec{F}_{e q}$ [35][36], that should produce the same deformation as the one produced by the free magnetostriction. In the variational form, nodal equivalent forces at node $i$ are expressed as in 28):

$$
F_{e q}^{v, i}=-\int_{\Omega}\left(\operatorname{grad}_{s}\left(\vec{v}^{i}\right): \mathbb{C}: \epsilon_{\mu}\right) \mathrm{d} \Omega
$$

with $\mathbb{C}$ the stiffness tensor of the medium, $\vec{v}^{i}$ a vectorial test function associated with the i-th component of the displacement (each component being discretized by a nodal scalar element function), $F_{e q}^{v, i}$ the nodal equivalent force of node $i$ in the direction of $\vec{v}$ and $\boldsymbol{g r a d}_{s}$ the symmetric gradient. The nodal equivalent force is then transformed into equivalent force density $\vec{f}_{\text {eq }}$, simply by dividing the force by the surface of the dual element associated with each node. This equivalent force density is then loaded as a body force of a pure mechanical problem.

\section{Mechanical feedback loop}

Magnetostriction induced deformations create stress fields that modify the magneto-mechanical behaviour of materials. This influence is usually considered negligible in power transformers due to the low magnetostriction of the materials that are employed. This magnetostriction is nevertheless not null, and can differ strongly from one layer to another at the same contact point. Because layers in the real transformer are assembled together, creating a uniform displacement and consequently a uniform deformation, the elastic deformation 
can strongly differ from one layer to another at the same point. The local stress can consequently vary strongly through the layers, even if its average value remains small. To the author's knowledge, such kind of configuration has never been tested. The mechanical feedback introduced in the loop in time domain is designed as an independent computation block in parallel with the magnetic loop. It can be activated in order to take the induced stress into consideration, or deactivated to economize the computation time.

In this mechanical feedback loop, the static mechanical resolution is carried out first, taking equivalent force density as mechanical loadings 29 . This leads to the total strain field $\epsilon$, sum of the elastic and free magnetostriction strains $(30)$, where $\boldsymbol{\epsilon}_{e l 1}\left(\boldsymbol{\epsilon}_{e l 2}\right)$ and $\boldsymbol{\epsilon}_{\mu 1}\left(\boldsymbol{\epsilon}_{\mu 2}\right)$ are respectively the elastic and free magnetostriction strains in layer family 1 (layer family 2 ).

$$
\begin{aligned}
\vec{f}_{e q} & =-\overrightarrow{\operatorname{div}} \tilde{\boldsymbol{\sigma}} \quad \text { with: } \tilde{\boldsymbol{\sigma}}=\mathbb{C}: \boldsymbol{\epsilon} \\
\boldsymbol{\epsilon} & =\boldsymbol{\epsilon}_{e l 1}+\boldsymbol{\epsilon}_{\mu 1}=\boldsymbol{\epsilon}_{e l 2}+\boldsymbol{\epsilon}_{\mu 2}
\end{aligned}
$$

Magnetostriction induced stress at the structure level for each layer is finally obtained by a simple linear elastic relation (31).

$$
\boldsymbol{\sigma}_{1}=\mathbb{C}: \boldsymbol{\epsilon}_{e l 1} \quad \text { and } \quad \boldsymbol{\sigma}_{2}=\mathbb{C}: \boldsymbol{\epsilon}_{e l 2}
$$

This stress is injected in the SMSM in the magnetic loop, leading to a fully coupled magneto-mechanical solution. In practice, the convergence procedure can be stabilized by the introduction of a relaxation coefficient.

\section{Harmonic mechanical resolution}

In order to avoid the transient computation which normally takes a huge amount of time, equivalent forces at each time step are computed in the frequency space using a Fast Fourier Transformation (FFT) method. This vibration problem is then considered as a purely harmonic linear elastic problem. Deformation and displacement fields at each harmonic are then obtained by applying (32) to each harmonic component of this equivalent force density $\vec{f}_{e q}^{n}$ as mechanical excitation, where $\omega^{n}$ is the pulsation of the $\mathrm{n}^{\text {th }}$ harmonic, $\beta$ is the damping coefficient and $\rho$ the mass density.

$$
\overrightarrow{\operatorname{div}} \tilde{\boldsymbol{\sigma}}^{n}+\vec{f}_{e q}^{n}=-\rho\left(\omega^{n}\right)^{2} \vec{u}^{n}+j \beta \rho\left(\omega^{n}\right) \vec{u}^{n}
$$

with: $\quad \tilde{\boldsymbol{\sigma}}^{n}=\mathbb{C}: \boldsymbol{\epsilon}^{n} \quad$ and $\quad \boldsymbol{\epsilon}^{n}=\boldsymbol{\epsilon}_{e l}^{n}+\boldsymbol{\epsilon}_{\mu}^{n}=\boldsymbol{g r a d}_{s} \vec{u}^{n}$

$\epsilon^{n}$ and $\epsilon_{e l}^{n}$ are the total and elastic strain of the $\mathrm{n}^{\text {th }}$ harmonic and $\epsilon_{\mu}^{n}$ the magnetostrictive one. The plane stress assumption is considered. An inverse Fourier Transformation is then applied, leading to the displacement of all nodes over the time.

\section{E. Acoustic power}

The displacement field is obtained for each harmonic from the mechanical resolution, leading to the estimation the vibration generation induced by core deformation. A block of posttreatment is next added, calculating the acoustic power $P_{a c}$. Acoustic power is an integration of the sound intensity $\vec{I}_{a c}$ along the external oriented surface of the considered structure $\vec{s}$ 34. $\vec{I}_{a c}^{n}$ is the $n^{t h}$ harmonic of sound intensity, which is the product of the $n^{\text {th }}$ sound pressure $P_{a c}^{n}$ and particule velocity $\vec{V}^{n}$ (effective value) 35 .

$$
\begin{gathered}
P_{a c}=\int_{s} \vec{I}_{a c} \mathrm{~d} \vec{s}=\int_{s} \sum_{n} \vec{I}_{a c}^{n} \mathrm{~d} \vec{s} \\
\vec{I}_{a c}^{n}=P_{a c}^{n} \vec{V}^{n}=Z_{0}\left(V^{n}\right)^{2} \vec{n}
\end{gathered}
$$

$\vec{n}$ is a unit vector of the particle velocity direction. $Z_{0}$ is the acoustic impedance, which equals $409.4 \mathrm{Pas} / \mathrm{m}$ at the room temperature in free space. Sound pressure $P_{a c}^{n}$ is expressed as: $P_{a c}^{n}=Z_{0} \vec{V}^{n}$. A strong advantage of the formulation is that the acoustic power is neither room-dependent nor distancedependent.

In this paper, the acoustic power is estimated using a fictive $3 \mathrm{D}$ structure extruded from the $2 \mathrm{D}$ simulation. The total surface can be computed, once the thickness of the transformer core is given. Out of plane component of the total strain $\epsilon_{z z}$ is obtained from the in-plane components $36 . \nu$ is the Poisson ratio.

$$
\epsilon_{z z}=\frac{1-2 \nu}{1-\nu} \epsilon_{z z}^{\mu}-\frac{\nu}{1-\nu}\left(\epsilon_{x x}+\epsilon_{y y}\right)
$$

From $\epsilon_{z z}$, the movement in direction $\vec{z}$ of the computation area is obtained. The contribution of this surface is then added to get the total acoustic power.

\section{Application to GO FeSi core}

GO FeSi is chosen as the magnetic material for this study because of its strong anisotropy. This material is processed in such a way that it offers better magnetic properties in its easy magnetization direction (rolling direction RD), due to a dominant quantity of domains oriented along this direction. On one hand, this material is the best example to verify the homogenization law and the correct implementation of the SMSM in the modeling chain. On the other hand, it may be a good candidate for this application if the advantages brought by the RD are higher than the drawbacks brought by the transversal direction (TD).

Fig 4 allow to compare the magnetic and magnetostrictive behaviors for a magnetic field applied along RD and TD, illustrating the strong anisotropy of GO material, and especially a very low magnetostriction amplitude along RD. SMSM parameters of GO are set according to experimental results [30] (given in table I]. SMSM gives magnetic and magnetostrictive behaviors under stress, with traction (positive value) and compression (negative value) stress applied in the direction of magnetic field. 1

1

- As the Grain-Oriented FeSi is very close to monocristal, the SMSM gives similar results compaired to the MSM.

- A comparson of modeling and measured material behavior, together with the parameters used in MSM, are given in [Hubert \& Daniel 2008 - JMMM].

- The behavior of the Grain-Oriented FeSi under stress, together with the results comparison of MSM and measurements is already done in [K.J. Rizzo, O.Hubert and L.Daniel, IEEE Transactions on Magnetics, 46, 2 (2010) 270-273.] 


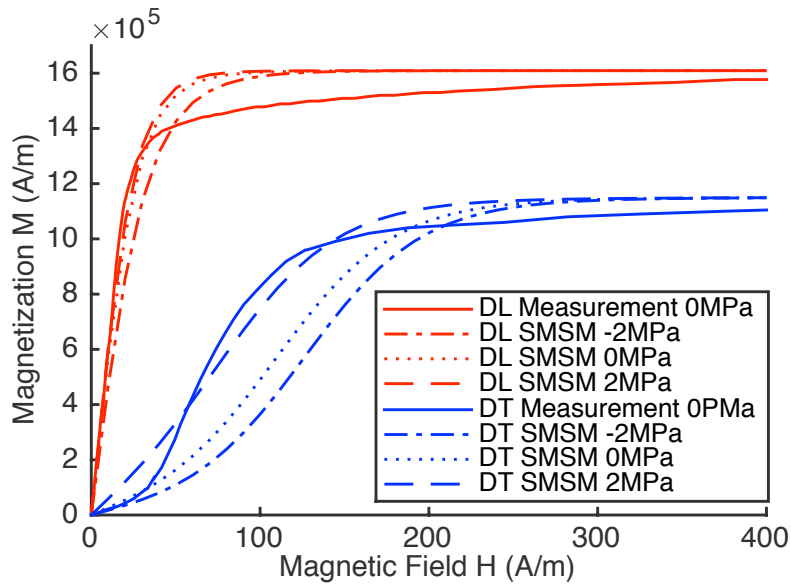

Fig. 4: Comparison of SMSM results (dots) to experimental measurement (lines) [30] - anhysteretic magnetization curves for magnetic field applied along RD (red) and TD (blue).
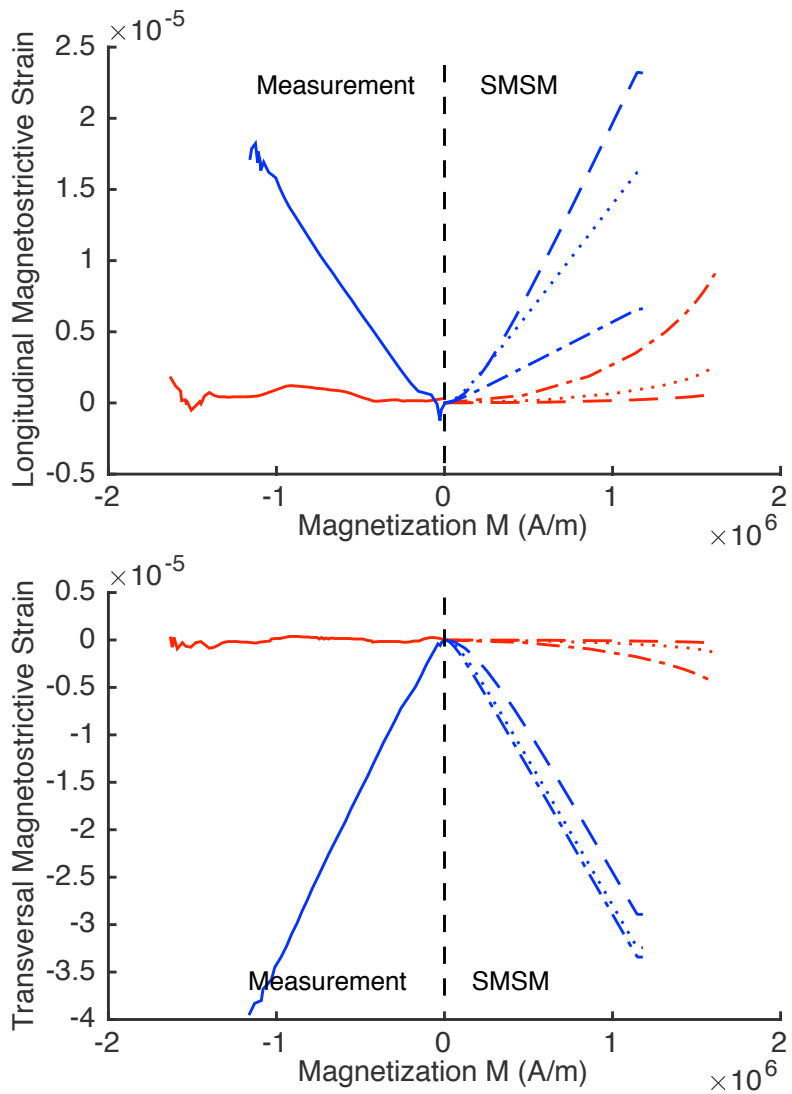

\begin{tabular}{|ll|}
\hline - DL Measurement OPMa & DT Measurement OPMa \\
\hdashline$\ldots \ldots$ - DL SMSM -2MPa & $\ldots \ldots \ldots$ DT SMSM -2MPa \\
$\ldots \ldots \ldots$ DL SMSM OMPa & $\ldots \ldots$ DT SM OMPa \\
- - DL SMSM 2MPa & $-\ldots$ DT SMSM 2MPa \\
\hline
\end{tabular}

Fig. 5: Comparison of SMSM results (dots) to experimental measurement (lines) [30] - anhysteretic longitudinal (top) and transversal (bottom) magnetostriction strain for magnetic field applied along RD (red) and TD (blue).

Transformer stacked core made of GO FeSi shown in Fig 2 is first modeled, with its reference dimensions $213 \mathrm{~mm} \times$ $202 \mathrm{~mm}$. The width of yokes $\left(W_{1}\right)$ and limbs $\left(W_{2}\right)$ is set as $40 \mathrm{~mm}\left(W_{1}=W_{2}=W=40 \mathrm{~mm}\right)$. Hundreds of layers are
TABLE I: Physical constants used for SMSM.

\begin{tabular}{|c|c|c|}
\hline Param. & FeSi GO & Unit \\
\hline \hline$M_{s}$ & $1.61 \times 10^{6}$ & $A / m$ \\
\hline$K_{1}$ & 38 & $\mathrm{~kJ} . \mathrm{m}^{-3}$ \\
\hline$\lambda_{100} ; \lambda_{111}$ & $23.5 ;-4.5$ & $\mathrm{ppm}$ \\
\hline$C$ & 5000 & ${\mathrm{~J} . m^{-3}}^{-3}$ \\
\hline$A_{s}$ & $20 \times 10^{-3}$ & $\mathrm{~m}^{3} / J$ \\
\hline
\end{tabular}

concerned, leading to a volume fraction of layer family 1 and 2 equal to each other $\left(f_{1}=f_{2}=0.5\right)$. A $40 \mathrm{~mm}$ transformer core thickness is supposed for the computation of the acoustic power at the border. All three phases are excited by sinusoidal magnetic flux (see Fig 2), with a phase-shift of $\frac{2 \pi}{3}$ from each other. The working frequency is set at $400 \mathrm{~Hz}$. Current in each phase is computed as a result of the magnetic resolution.

The finite element problems are solved using FreeFem++ [37] including the SMSM as a dynamically linked and parallelized function. Computation is processed on a personal computer of 8 cores with a clock $3.2 \mathrm{GHz}$. Fig 6 shows the current in the three phases as function of time for a magnetic flux excitation corresponding to a maximal induction of $B_{\max }=1.2 \mathrm{~T}$. Displacement field is shown in Fig 7 at instants $t_{1}$ and $t_{2}$ (indicated in Fig 6) as examples. $t_{1}$ is the initial computation step, $t_{2}$ corresponds to $\frac{1}{8}$ period. The deformed core shape is shown with a scale factor of 20000 for better visibility.

The constitutive behavior of materials is fully coupled. It means that some stress fields do exist and that they influence the overall behavior of the structure. Stress components of layer family $1\left(\sigma_{1 x x}, \sigma_{1 y y}\right.$ and $\left.\sigma_{1 x y}\right)$ and layer family 2 $\left(\sigma_{2 x x}, \sigma_{2 y y}\right.$ and $\left.\sigma_{2 x y}\right)$ are shown respectively in Fig 8 and Fig 9 In this example, they are given at instant $t_{2}$. We observe first that the stress levels reached for the two layer families are the same but extremal values are positioned symmetrically with respect to the $\mathrm{x}$ axis. Indeed the two layer families are geometrically symmetric with respect to this axis and they are considered at the same volume fraction for the calculation. We observe secondly that the stress level is relatively small in most of the areas except for some specific regions:

1) regions of connections between the E-shaped and Ishaped sheets present some stress concentrations especially for components $\sigma_{1 x x}$ and $\sigma_{2 x x}$ (up to $1.3 \mathrm{MPa}$ ). These stress concentrations are associated with the 90 degrees rotation of the easy direction, creating large magnetostriction variations.

2) some parts of the yokes undergo homogeneous stress along $\mathrm{Y}$ direction $\left(\boldsymbol{\sigma}_{1 y y}\right.$ and $\left.\boldsymbol{\sigma}_{2 y y}\right)$ reaching about $2.4 M P a$ of magnitude. These stresses are due to the difference in magneto-mechanical properties between TD and RD layers deposited alternately.

3) shear stresses are especially significant at the internal corners of the structure (reaching $\pm 0.3 \mathrm{MPa}$ ).

It must be, on the other hand, underlined that this stress distribution is changing during an excitation period. Plots are given at time $\mathrm{t} 2$ and are different at other times. Moreover 


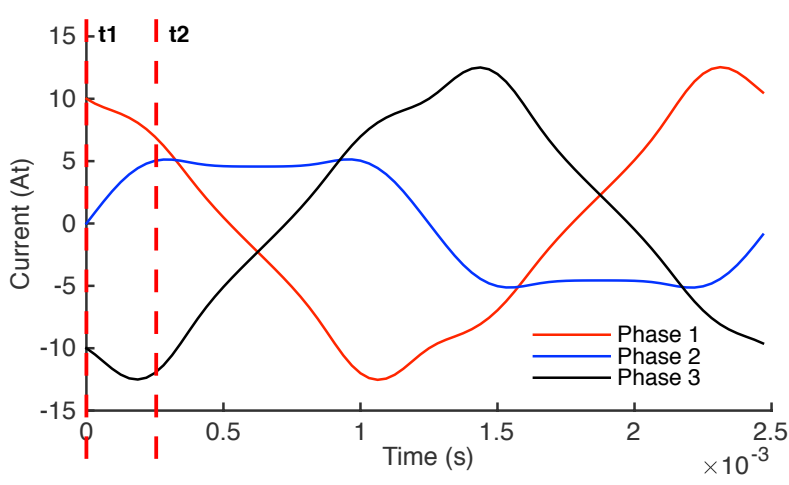

Fig. 6: Current in the three phases of one period of time.

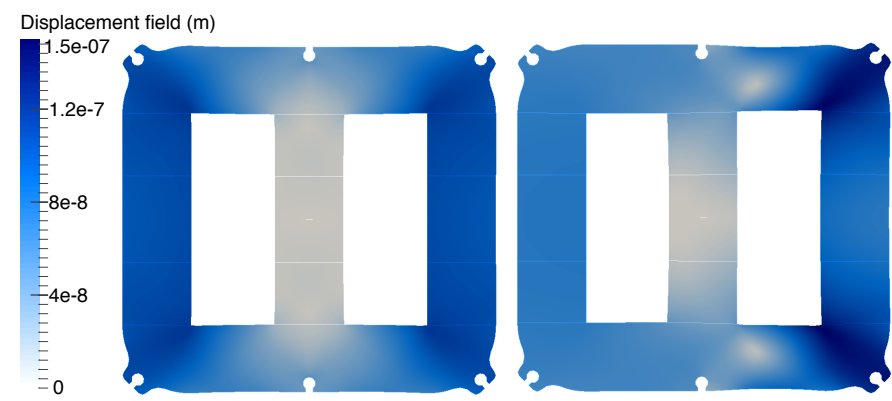

Fig. 7: Displacement field described by color density at $t_{1}$ (left) and $t_{2}$ (right). Deformed core shape is shown with a scale factor of 20000

even if the stress level is not high, previous works have demonstrated that the magnetic and magnetostrictive behavior of GO materials are strongly stress sensitive even at a low level [38]. The shear stress concentration at the internal corners could finally have a significant effect because it is positioned where the highest values of the magnetic flux densities are located. The numerical calculation will allow to appreciate the global effect of stress. It must be noticed that the calculation of a magneto-mechanical equivalent stress would be possible following [39] in order to appreciate the global effect of the stress field without any huge numerical implementation.

The root mean square (RMS) of the current in phase 1, 2 and 3 is respectively $7.98,4.29$ and 7.98 Ampere-turn, under no-load condition. This non-balanced current typical for a three-phase three-limb power transformer, because the magnetic reluctance for each phase is different. Average RMS of three phases is 6.97 Ampere-turn, defined as $I_{R M S(\operatorname{avg})}=$ $\sqrt{\left(I_{R M S 1}^{2}+I_{R M S 2}^{2}+I_{R M S 3}^{2}\right) / 3}$ which directly relates to energy loss by Joule effect. The shape of the current is deformed comparing to an ideal sinusoidal form. This is due to the non-linear permeability, and the interaction between phases. The total harmonic distortion (THD) describing the harmonic distortion is defined as the ratio of the sum of the amplitude of all harmonic components to the amplitude of the fundamental frequency:

$$
T H D=\frac{\sqrt{I_{2 n d}^{2}+I_{3 r d}^{2}+I_{4 t h}^{2}+\ldots}}{I_{1 s t}}
$$

THD of phases 1st, 2nd and 3rd are respectively 10.1\%, 27.9\% and $10.1 \%$.

To study the effect of mechanical feedback loop, a test computation is carried out using weakly coupled model (mechanical feedback loop deactivated) as comparison, with the magnetic flux excitation discretized in 80 steps. The comparison is summed up in table II. Fully coupled model leads to more current, higher current distortion and more acoustic power compared to weakly coupled model, showing clearly that the stresses degrade the material properties. However, the influence of this induced stress is lower than $10 \%$ in general. On an 8 -core $\mathrm{CPU}$ with a clock $3.2 \mathrm{GHz}$, a single computation using strongly coupled model takes about 20 hours, depending on the magnetic loadings and other settings. However, the simulation using a weakly coupled model only takes about 7 hours, leading to a simulation result similar enough to the strongly coupled one. In order to make the optimization process possible, the optimization process presented in the next section is carried out using the weakly coupled model.

TABLE II: Comparison of fully coupled and weakly coupled model.

\begin{tabular}{|c|c|c|c|}
\hline & $\begin{array}{l}\text { Fully } \\
\text { coupled }\end{array}$ & $\begin{array}{l}\text { Weakly } \\
\text { coupled }\end{array}$ & Unit \\
\hline \hline$I_{R M S(a v g)}$ & 6.97 & 6.66 & $A t$ \\
\hline$T H D$ phase 1,3 & 10.1 & 8.3 & $\%$ \\
\hline$T H D$ phase 2 & 27.9 & 23.7 & $\%$ \\
\hline$P_{a c}$ & 1.63 & 1.56 & $\mu W$ \\
\hline Computation time & 3.30 & 1.45 & $\mathrm{hrs}$ \\
\hline
\end{tabular}

\section{OPTIMIZATION OF CORE GEOMETRY}

As shown in Fig 7 , core deformation is mainly concentrated at the yokes where the magnetic field go from the rolling direction to the transversal direction (mixed zone), leading to huge magnetostriction induced deformation. Intuitively, one might think that increasing the width of the yokes and decreasing the width of the limbs in order to keep a constant mass would lead to an interesting solution to reduce the noise. Indeed, such transformation allows a new distribution of flux density: flux density is increased for parts along RD that exhibit a low magnetostriction; flux density is decreased for parts along TD that exhibit a high magnetostriction, leading to the reduction of the global deformation of the system. The initial width of yokes and limbs is $W$. In order to verify this pre-concidered idea, the new width of the yokes is defined by: $W_{1}=\alpha W$, with $\alpha$ a geometry coefficient. The width of the limbs is recalculated, in order to keep the mass and winding surface constant. Some examples of modified geometries are illustrated in Fig 10 for various $\alpha$.

A series of simulations is carried out with imposed maximum magnetic flux density from $1.2 T$ to $1.5 T$, for $\alpha$ varying from 0.8 to 1.2 . In the case of excitation $1.5 T$ at $\alpha=1.0$, the magnetic saturation is reached in limbs. No numerical solution exists for thinner limbs. Indeed, it is not possible to impose magnetic flux density higher than saturation. Average RMS and THD of current are shown in Fig 12 and Fig 13 


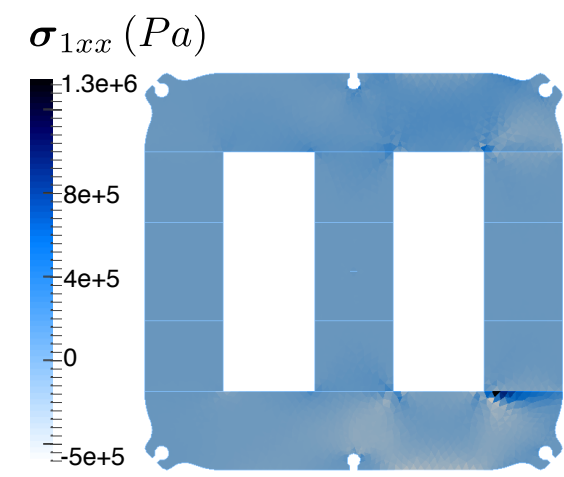

(a)

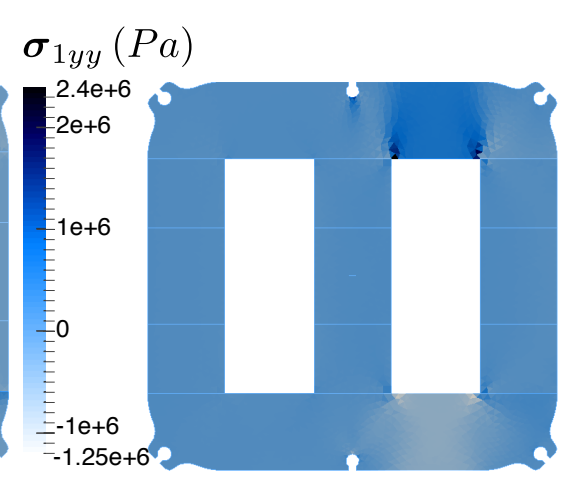

(b)

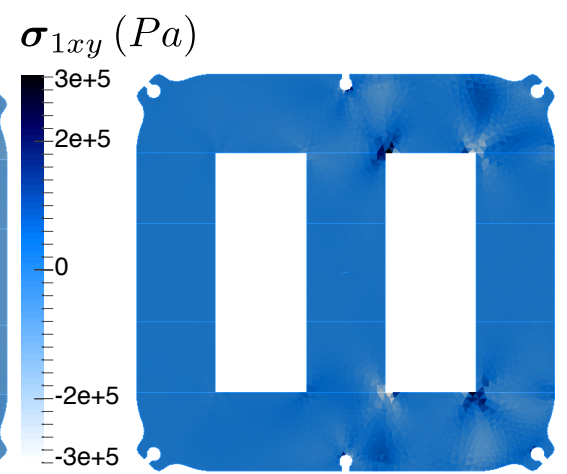

(c)

Fig. 8: Induced stress in-plane components for layer family 1 at instant $t_{2}$

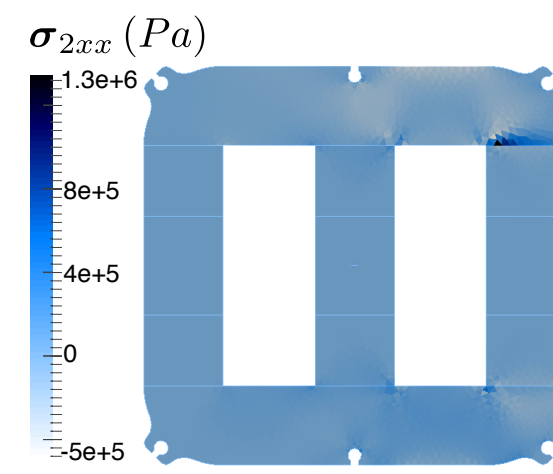

(a)

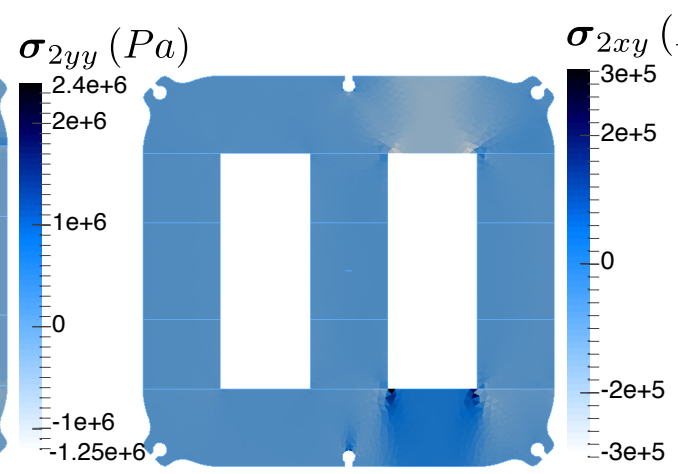

(b)

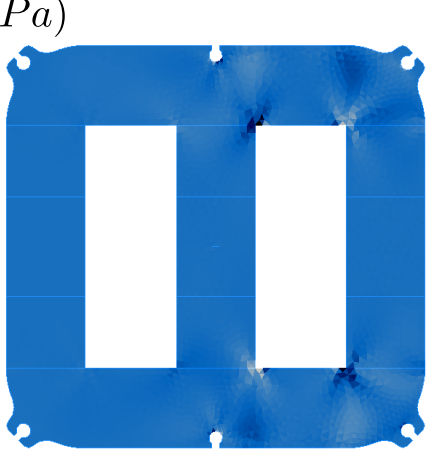

(c)

Fig. 9: Induced stress in-plane components for layer family 2 at instant $t_{2}$

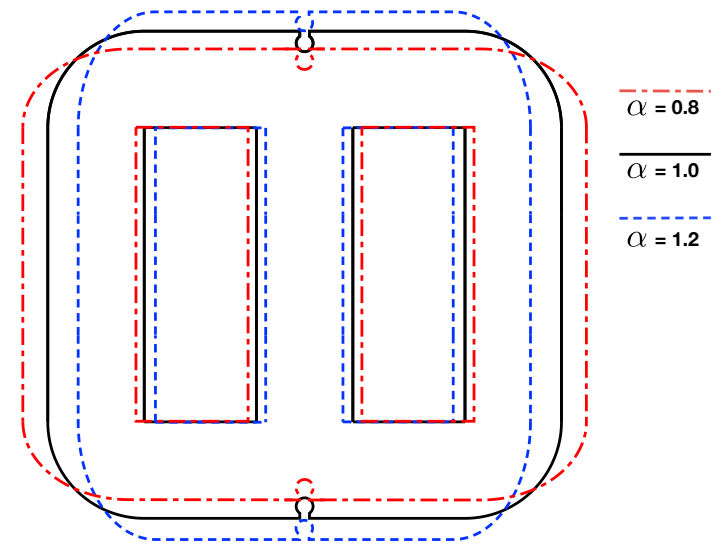

Fig. 10: Examples of modified geometry

As the magnetic flux is imposed with a sine waveform, nonlinearity and saturation of the material influence directly the amplitude and distortion of excitation current. At low magnetic flux excitation, a smaller current is enough to magnetize the transformer core. At high magnetic flux excitation, average RMS and THD increase rapidly with the flux excitation. By decreasing the geometry coefficient $\alpha$ (limbs wider and yokes thinner), average RMS and THD of the current increase dramatically because of the saturation in yokes. From an electric point of view, the optimized design of transformer core is obtained for a geometry coefficient close to $\alpha=1.05$ at high magnetic excitation, where average RMS and THD of current are both minimum. At low magnetic excitation, the average RMS and THD of current continue to decrease as $\alpha$ increases. Optimum point for low magnetic excitation may be higher than $\alpha=1.2$. This leads to the lowest joule losses.

The acoustic power is shown in Fig 14. The acoustic power is an interesting indicator of global deformation level, that may be related to the sound emission. At a given maximum flux density, the acoustic power varies with the geometry coefficient $\alpha$.

For high $\alpha$, the width of the yokes is larger than that of the limbs, leading to low flux density and then low deformation in yokes, where magnetic flux lines up to TD and normally creates large deformation. As the $\alpha$ goes extremely high, the deformation in limbs becomes larger because of the saturation. For low $\alpha$, the width of the yokes is smaller than that of the limbs, so that saturation first appears in yokes, leading to a huge deformation. On the other hand, the optimum $\alpha$ corresponding to the minimum acoustic power changes with the magnetic induction. For $B=1.2 T$ at $\alpha=1.0$, optimum $\alpha$ locates at 1.1 , which means yokes wider than limbs. This verifies the speculation made previously that by increasing the width of yokes and decreasing the induction in the transversal direction of the lamination, the noise can be reduced. As the 

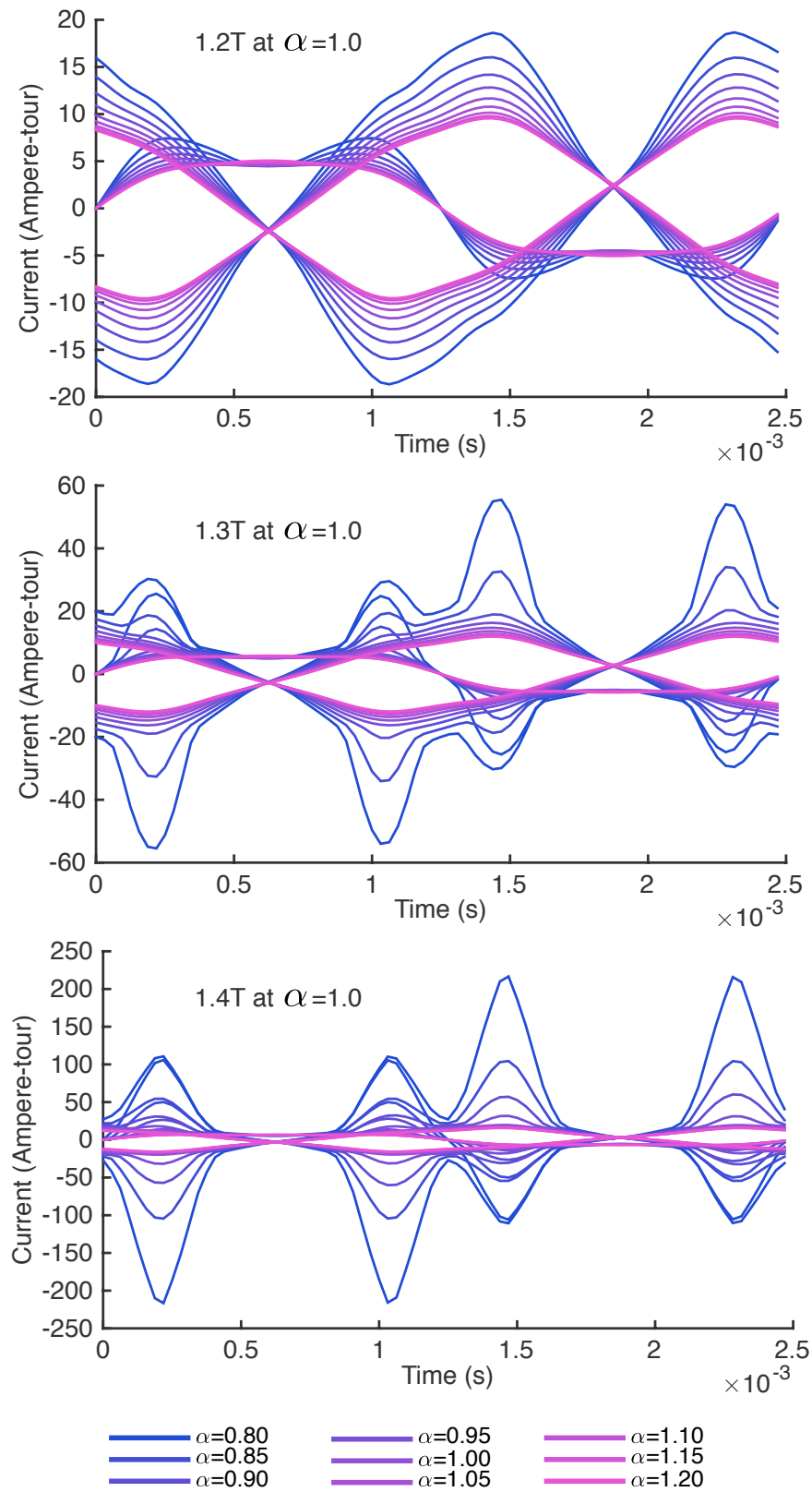

Fig. 11: Current of three phases with maximum induction of $1.2 T$ (top), $1.3 T$ (middle) and $1.4 T$ (bottom) on different geometries.

magnetic flux increases, the optimum $\alpha$ decreases. It reaches 1.0 at $B=1.5 T$. At this high level of excitation, where all areas of the transformer core are almost saturated, acoustic power is no more sensitive to the geometry variations. Other mechanisms coming from assembly precision and air gaps may also have an influence on the sound emission but are not considered in this simulation. It has been verified that the optimized geometry remains roughly the same when the fully coupled SMSM is used.

\section{CONCLUSION}

This complete fully coupled modeling chain, from three phase magnetic flux injection to the $2 \mathrm{D}$ deformation and

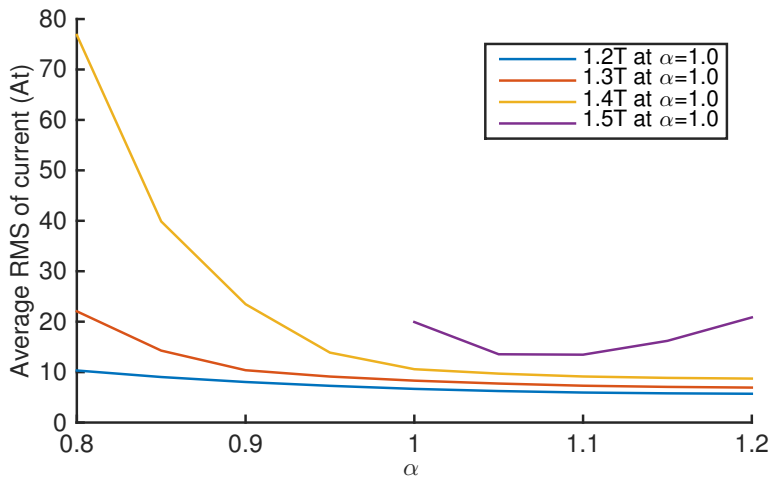

Fig. 12: Average RMS of current of three phases with different maximum inductions and geometries.

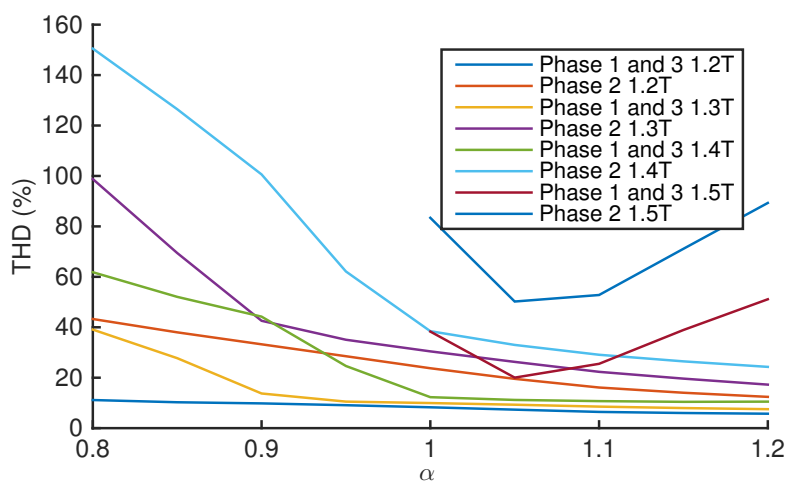

Fig. 13: THD with different maximum inductions and geometries.

acoustic power estimation of the transformer core, gives great practicality for transformer core design. Phase current coupling, magnetic flux excitation (voltage excitation), material anisotropy, magnetostriction induced stress and multi-layer homogenization are taken into account, leading to an easy comparison of different core structures and materials. Optimization of geometry can be carried out considering electric and acoustic aspects. For a GO FeSi 'E-I' transformer, it has been shown that the geometry coefficient $\alpha$ should be set to a value between 1.0 and 1.1 depending on the excitation level to reduce the acoustic emission.

\section{REFERENCES}

[1] M. Goto, H. Tange, T. Kamimori, and K. Okuma, "Saturation magnetostriction of some (FeCo)-metalloid-compounds and -amorphous alloys," Journal of Magnetism and Magnetic Materials, vol. 31-34, no. PART 3, pp. 1601-1602, 1983.

[2] T. Burkert, L. Nordström, O. Eriksson, and O. Heinonen, "Giant magnetic anisotropy in tetragonal FeCo alloys," Physical review letters, vol. 93, no. 2, p. 27203, 2004.

[3] T. Sourmail, "Near equiatomic FeCo alloys: Constitution, mechanical and magnetic properties," pp. 816-880, 2005.

[4] B. D. Cullity and C. D. Graham, "Magnetostriction and the Effects of Stress," in Introduction to Magnetic Materials, 2009, pp. 241273. [Online]. Available: http://ieeexplore.ieee.org/xpl/articleDetails. jsp?arnumber $=5488895$

[5] R. M. Bozorth, "Ferromagnetism," Ferromagnetism, by Richard M. Bozorth, pp. 992. ISBN 0-7803-1032-2. Wiley-VCH, August 1993., vol. 1, 1993. 


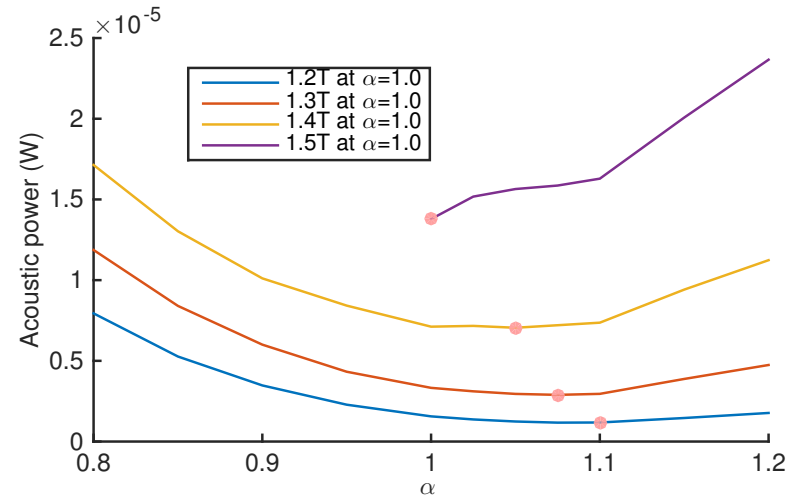

Fig. 14: Acoustic power with different maximum flux density and geometries.

[6] M. Ertl and H. Landes, "Investigation of load noise generation of large power transformer by means of coupled 3D FEM analysis," COMPEL, vol. 26 , no. 3, pp. $788-799,2007$.

[7] E. Du Trémolet de Lacheisserie and E. D. T. De Lacheisserie, Magnetostriction. CRC Press, 1993.

[8] B. Weiser and H. Pfützner, "Relevance of magnetostatic forces for transformer core vibrations," Le Journal de Physique IV, vol. 8, no. 1998 , pp. 8-11, 1998.

[9] M. Liu, O. Hubert, X. Mininger, F. Bouillault, and L. Bernard, "Homogenized magneto-elastic behavior model for the computation of strain due to magnetostriction in transformers," IEEE Transactions on Magnetics, vol. 52, no. 2, pp. 1-12, 2016.

[10] A. Shahaj and S. D. Garvey, "A possible method for magnetostrictive reduction of vibration in large electrical machines," IEEE Transactions on Magnetics, vol. 47, no. 2, pp. 374-385, feb 2011.

[11] L. Zhu, B. Wang, R. Yan, Q. Yang, Y. Yang, and X. Zhang, "Electromagnetic Vibration of Motor Core Including Magnetostriction Under Different Rotation Speeds," IEEE Transactions on Magnetics, vol. 52, no. 3, pp. 1-4, mar 2016.

[12] M. Rossi and J. Le Besnerais, "Vibration reduction of inductors under magnetostrictive and Maxwell forces excitation," IEEE Transactions on Magnetics, vol. 51, no. 12, pp. 1-6, dec 2015.

[13] M. Mizokami and Y. Kurosaki, "Noise variation by compressive stress on the model core of power transformers," Journal of Magnetism and Magnetic Materials, vol. 381, pp. 208-214, may 2015.

[14] L. Zhu, Q. Yang, R. Yan, Y. Li, X. Zhang, W. Yan, and J. Zhu, "Numerical computation for a new way to reduce vibration and noise due to magnetostriction and magnetic forces of transformer cores," Journal of Applied Physics, vol. 113, no. 17, pp. 2014-2017, 2013.

[15] C.-H. Hsu, C.-Y. Lee, S.-J. Cheng, C.-M. Fu, and C.-W. Chang, "Effects of magnetostriction and magnetic reluctances on magnetic properties of distribution transformers," Journal of Applied Physics, vol. 115, no. 17, p. 17E718, may 2014

[16] S.-J. Cheng, J.-J. Liu, Y.-H. Chang, C.-M. Fu, C.-H. Hsu, C.-Y. Lee, and C.-W. Chang, "Correlation of magnetostriction variation on magnetic loss and noise for power transformer," Journal of Applied Physics, vol. 117, no. 17, p. 17E716, 2015.

[17] J. Wang, C. Gao, X. Duan, and K. Mao, "Multi-field coupling simulation and experimental study on transformer vibration caused by DC bias," Journal of Electrical Engineering and Technology, vol. 10, no. 1, pp. 176-187, jan 2015.

[18] P. Witczak, "Magnetostriction force spectrum in power transformer," Electrical Machines (ICEM), 2014 International Conference, no. 3, pp. 2246-2251, 2014.

[19] S. G. Ghalamestani, L. Vandevelde, and J. Melkebeek, "Identification of transformer core vibrations and the effect of third harmonic in the electricity grid," International Journal of Electrical, Computer, Energetic, Electronic and Communication Engineering, vol. 8, no. 6, pp. 871-874, 2014.

[20] C.-h. Hsu, S.-1. Lee, C.-c. Lin, C.-s. Liu, S.-y. Chang, M.-f. Hsieh, Y.m. Huang, and C.-m. Fu, "Reduction of vibration and sound-level for a single-phase power transformer with Large capacity," IEEE Transactions on Magnetics, vol. 51, no. 11, pp. 1-4, nov 2015.

[21] T. Hilgert, L. Vandevelde, and J. Melkebeek, "Comparison of magnetostriction models for use in calculations of vibrations in magnetic cores," IEEE Transactions on Magnetics, vol. 44, no. 6, pp. 874-877, 2008.

[22] P. Shuai and J. Biela, "Investigation of acoustic noise sources in medium frequency, medium voltage transformers," in 2014 16th European Conference on Power Electronics and Applications, aug 2014, pp. 1-11.

[23] _ - "Impact of core shape and material on the acoustic noise emission of medium frequency, medium voltage transformers," in 2015 17th European Conference on Power Electronics and Applications (EPE'15 ECCE-Europe). IEEE, sep 2015, pp. 1-11.

[24] C. H. Hsu, M. F. Hsieh, C. M. Fu, and Y. M. Huang, "Effects of Multicore Structure on Magnetic Losses and Magnetomechanical Vibration at High Frequencies," IEEE Transactions on Magnetics, vol. 51, no. 11, pp. 3-6, 2015.

[25] C. H. Hsu, J. J. Liu, C. M. Fu, Y. M. Huang, C. W. Chang, and S. J. Cheng, "Suppressing magneto-mechanical vibrations and noise in magnetostriction variation for three-phase power transformers," Journal of Applied Physics, vol. 117, no. 17, 2015.

[26] Y. Yang, L. Qingfen, L. Dichen, L. Shanshan, and H. Jingzhu, "Electromagnetic vibration noise analysis of transformer windings and core," IET Electric Power Applications, vol. 10, no. 4, pp. 251-257, apr 2016.

[27] A. Belahcen, D. Singh, P. Rasilo, F. Martin, S. G. Ghalamestani, and L. Vandevelde, "Anisotropic and strain-dependent model of magnetostriction in electrical steel sheets," IEEE Transactions on Magnetics, vol. 51, no. 3, pp. 1-4, mar 2015.

[28] S. Mbengue, N. Buiron, and V. Lanfranchi, "An anisotropic model for magnetostriction and magnetization computing for noise generation in electric devices," Sensors, vol. 16, no. 4, p. 553, apr 2016.

[29] D. Vanoost, S. Steentjes, H. De Gersem, J. Peuteman, G. Gielen, D. Pissoort, and K. Hameyer, "Embedding a magnetoelastic material model in a coupled magnetomechanical finite-element solver," IEEE Transactions on Magnetics, vol. 51, no. 11, pp. 1-4, nov 2015.

[30] O. Hubert and L. Daniel, "Multiscale modeling of the magnetomechanical behavior of grain-oriented silicon steels," Journal of Magnetism and Magnetic Materials, vol. 320, no. 7, pp. 1412-1422, apr 2008.

[31] L. Daniel, O. Hubert, N. Buiron, and R. Billardon, "Reversible magnetoelastic behavior: a multiscale approach," Journal of the Mechanics and Physics of Solids, vol. 56, no. 3, pp. 1018-1042, mar 2008.

[32] L. Daniel, "An analytical model for the effect of multiaxial stress on the magnetic susceptibility of ferromagnetic materials," IEEE Transactions on Magnetics, vol. 49, no. 5, pp. 2037-2040, may 2013.

[33] L. Bernard and L. Daniel, "Effect of stress on magnetic hysteresis losses in a switched reluctance motor: application to stator and rotor shrinkfitting," IEEE Transactions on Magnetics, vol. 51, no. 9, pp. 1-1, 2015.

[34] S. Bouissou and F. Piriou, "Study of 3D formulations to model electromagnetic devices," IEEE Transactions on Magnetics, vol. 30, no. 5, pp. 3228-3231, 1994.

[35] N. Galopin, X. Mininger, F. Bouillault, and L. Daniel, "Finite element modeling of magnetoelectric sensors," IEEE Transactions on Magnetics, vol. 44, no. 6, pp. 834-837, jun 2008.

[36] D. J. Payen and K. J. Bathe, "The use of nodal point forces to improve element stresses," Computers and Structures, vol. 89, no. 5-6, pp. 485495, 2011.

[37] F. Hecht, "New development in freefem ++," Journal of Numerical Mathematics, vol. 20, no. 3, pp. 251-265, 2012

[38] K. J. Rizzo, O. Hubert, and L. Daniel, "Magnetic and magnetostrictive behavior of iron-silicon single crystals under uniaxial stress," IEEE Transactions on Magnetics, vol. 46, no. 2, pp. 270-273, 2010.

[39] O. Hubert and L. Daniel, "Energetical and multiscale approaches for the definition of an equivalent stress for magneto-elastic couplings," Journal of Magnetism and Magnetic Materials, vol. 323, no. 13, pp. 1766-1781, 2011. 